\title{
Combining frequency and time domain approaches to systems with multiple spike train input and output
}

\author{
D. R. Brillinger · K. A. Lindsay · J. R. Rosenberg
}

Received: 15 July 2008 / Accepted: 18 December 2008 / Published online: 30 January 2009

(C) The Author(s) 2009. This article is published with open access at Springerlink.com

\begin{abstract}
A frequency domain approach and a time domain approach have been combined in an investigation of the behaviour of the primary and secondary endings of an isolated muscle spindle in response to the activity of two static fusimotor axons when the parent muscle is held at a fixed length and when it is subjected to random length changes. The frequency domain analysis has an associated error process which provides a measure of how well the input processes can be used to predict the output processes and is also used to specify how the interactions between the recorded processes contribute to this error. Without assuming stationarity of the input, the time domain approach uses a sequence of probability models of increasing complexity in which the number of input processes to the model is progressively increased. This feature of the time domain approach was used to identify a preferred direction of interaction between the processes underlying the generation of the activity of the primary and secondary endings. In the presence of fusimotor activity and dynamic length changes imposed on the muscle, it was shown that the activity of the primary and secondary endings carried different information about the effects of the inputs imposed
\end{abstract}

This article is part of a special issue on Neuronal Dynamics of Sensory Coding.

D. R. Brillinger

Department of Statistics, University of California,

Berkeley, CA 94720-3860, USA

K. A. Lindsay

Department of Mathematics,

University Gardens, University of Glasgow,

Glasgow G12 8QW, UK

J. R. Rosenberg ( $\varangle)$

Division of Neuroscience and Biomedical Systems,

University of Glasgow, Glasgow G12 8QQ, UK

e-mail: j.rosenberg@bio.gla.ac.uk on the muscle spindle. The results presented in this work emphasise that the analysis of the behaviour of complex systems benefits from a combination of frequency and time domain methods.

Keywords Multiple spike trains - Neural coding . Maximum likelihood - Canonical coherence $\cdot$ Muscle spindle $\cdot$ Multivariate point processes

\section{Introduction}

The behaviour of networks of neurons is commonly described in terms of the strength of association between pairs of neurons within the network, an assessment of their timing relations and the identification of those neurons which interact directly or are influenced by common inputs. Consistent with the view presented by Segundo (Cox et al. 1975), what is required is an approach which characterises the behaviour of a network as a single object as opposed to that of pairwise measures.

Pepe Segundo, beginning with his work on aplysia (see, Moore this issue), worked at the interface between Neuroscience and Statistics and continued this type of work for the majority of his career. The work presented here contributes to that endeavour and at the same time illustrates that work of this kind will necessarily contribute to both fields. We shall outline two approaches for analysing the behaviour of networks of neurons based on a generalised linear model relating vectors of input and output processes in which the components of these vectors can be either continuous or point process signals.

The first model is formulated in terms of Fourier transforms, and its performance is described by how well it predicts output network behaviour. It is shown that the spectral density matrix of the output processes can be decomposed 
Fig. 1 Diagrammatic representation of a muscle spindle illustrating the three types of intrafusal muscle fibre $\left(D b_{1}\right.$ dynamic bag $_{1}, S b_{2}$ static bag $_{2}$ and chain fibres), their motor innovation ( $\gamma$-dynamic and $\gamma$-static fusimotor axons), and the primary $(I a)$ and secondary (II) sensory endings. The small inserts in Figs. 3 and 4 may be interpreted in terms of this figure

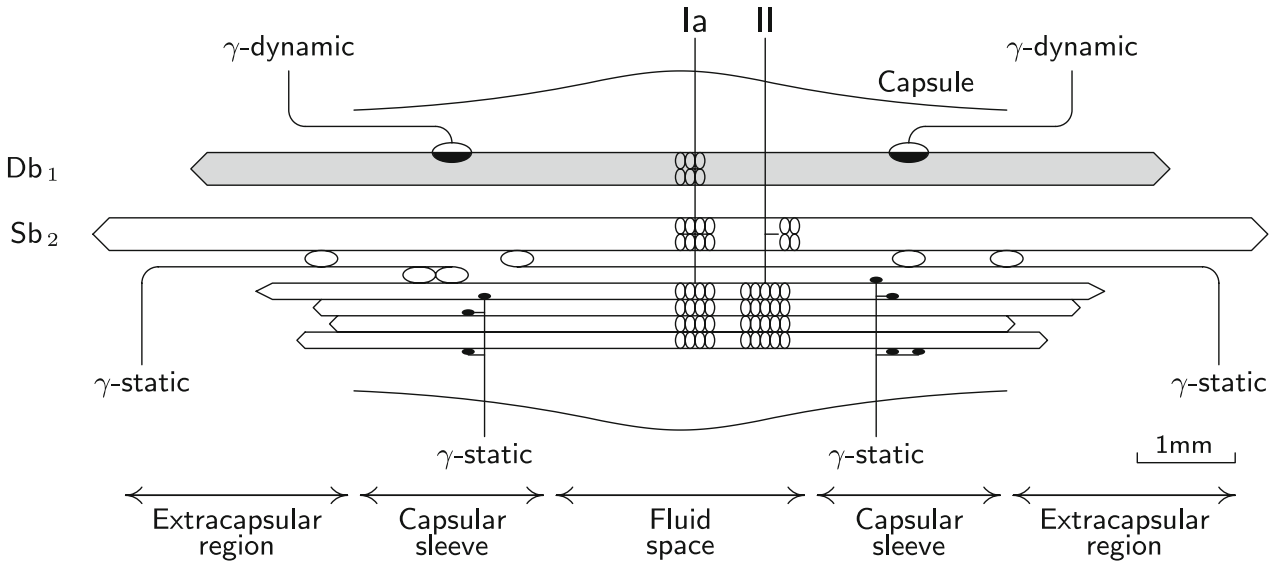

into a series of spectral density matrices. The sum of these matrices, each weighted by a factor which may be interpreted as a generalised coherence, forms the spectral density matrix of the error process for the network model. The measure of size of the error process is taken to be the largest eigenvalue (Hermitian norm) of the spectral density matrix of the output process. Departures of the Hermitian norm of the error process from its maximum value provides a measure of the adequacy of the linear model. The causes for these departures can be explained by the behaviour of the eigenvalues in the expansion of the spectrum of the error process. The structure of these eigenvalues reflects the influence of connectivity within the network.

The second model is based on the complete intensity function of the output processes given the history of the input and output processes. This model is an adaptation of that used in Brillinger and Segundo (1979) and Brillinger (1992), but applied to the mammalian muscle spindle under conditions of multiple input and multiple output. The estimates of the parameters of this model are based on a likelihood function and therefore may be anticipated to be reasonably efficient. Further, these estimates may be expected to highlight different aspects of the system behaviour than those highlighted by the Fourier method. An advantage of the second approach is that the input can be nonstationary and other explanatory variables introduced directly.

Whereas the first model examines the global behaviour of the system, the second model characterises the interactions among the recorded processes by constructing a sequence of generalised linear models of increasing complexity progressing from single input single output to multiple input multiple output models in which the complete intensity function is now formulated in terms of vector-valued processes. The two approaches to network behaviour described here are illustrated by the analysis of an experiment on an isolated mammalian muscle spindle in which the data from two input point processes and two output point process are available for analysis. A particular feature of the analysis is that the association of the activity between primary and secondary muscle spindle endings is thought to be functionally important (Gladden and Matsuzaki 2002; Halliday et al. 1987). For example, interneurons in di-synaptic excitatory and some inhibitory pathways from group I and group II afferents receive inputs from both primary and secondary muscle spindle axons (Edgley and Jankowska 1987; Jankowska 1992; Jankowska and Hammar 2002). The effects that these interneurons exert on the $\alpha$-motoneurons would be facilitated by associations induced between the activity of primary and secondary muscle spindle endings controlled by fusimotor activity and length changes imposed on the muscle spindle. The changes in correlation between the discharges of the primary and secondary endings can best be examined when these discharges are recorded from the same muscle spindle. In this case the effect of the controlling processes can be associated directly with the pattern of fusimotor innervation of the muscle spindle.

\section{Isolated mammalian muscle spindle data}

The examples presented in both the frequency and time domain analyses in this work are based on an experiment in which the primary (Ia) and secondary endings (II) from the same muscle spindle are isolated in dorsal root filaments (Gladden and Matsuzaki 2002; Halliday et al. 1987). Single static fusimotor axons $(\gamma)$ were isolated in cut ventral root filaments of cat spinal chord. Figure 1 provides a diagrammatic representation of a muscle spindle illustrating the three types of intrafusal muscle fibres along with their motor (static and dynamic fusimotor axons) and primary (Ia) and secondary (II) sensory axons.

Detailed descriptions of the distribution of the motor and sensory axons to the three types of intrafusal muscle fibre are given by Banks et al. (1981), whereas Boyd (1981) describes the response of the primary and secondary endings to the separate activation of each type of intrafusal muscle fibre.

The responses of the primary and secondary endings were recorded simultaneously during stimulation of one or 
both of the two identified static gamma axons in the presence and absence of concomitant random length changes. The fusimotor axons were stimulated by pulses with a homogeneous exponential distribution of independent intervals, that is a Poisson process. In some trials length changes with a Gaussian distribution of amplitudes were imposed on the parent muscle (tenuissimus) with simultaneous stimulation of the fusimotor axons. The imposed length changes were selected to have a constant spectral density over the range zero to $100 \mathrm{~Hz}$.

The data sets consist of 60-s records of simultaneously recorded $\gamma_{1}, \gamma_{2}$, Ia and II spike train activity in which spikes are isolated to an interval of $1 \mathrm{~ms}$ duration. The results of the analysis are based on the one experiment in which the primary and secondary endings are isolated from the same muscle spindle. It is not intended that these results are to be generalised to all muscle spindles since it is the objective of this article to present a methodology suitable for the analysis of any system with multiple inputs and output processes, and to initiate a discussion of the biological implications of such results. The estimation procedures used in the frequency domain approach are set out in Rosenberg et al. (1989). Partial spectra were used to identify a network of neurons as in Brillinger et al. (1976). In summary, the spectra are estimated by dividing each 60 -s record into 58 contiguous intervals of $1,024 \mathrm{~ms}$ and the estimates of the spectra are taken to be the average of the periodograms computed for each interval. The parameters in the time domain analysis are estimated for a maximum lag of $50 \mathrm{~ms}$ using the $\mathrm{R}$ integrated suite of software facilities (CRAN 2008).

\section{A frequency domain approach}

Let $\mathcal{M}$ and $\mathcal{N}$ be stationary and orderly vector point processes satisfying a mixing condition. The orderly property states that events of $\mathcal{M}$ and $\mathcal{N}$ are simple/isolated and the mixing property states that events well separated in time are approximately independent. The approach to spiking neural networks taken in this section is based on the development of the multi-dimensional point process linear model proposed by Brillinger (1975a) and draws upon the properties of the finite Fourier transform of a stationary process (Brillinger 1983). In this model the instantaneous vector of rates $\mu_{\mathcal{N}}(t)$ of the output point processes $\mathcal{N}$ is related to the input point process $\mathcal{M}$ by the equation

$$
\begin{aligned}
\mu_{\mathcal{N}}(t) & =\lim _{h \rightarrow 0+} \frac{\operatorname{Prob}\{\text { An } \mathcal{N} \text {-event in }[t, t+h) \mid \mathcal{M}\}}{h} \\
& =\mu+\int_{-\infty}^{\infty} K(t-u) \mathrm{d} M(u)
\end{aligned}
$$

where $\mu$ is a constant vector, $K$ is a time-varying matrix ${ }^{1}$ with row and column dimensions the number of processes in the vectors $\mathcal{N}$ and $\mathcal{M}$ respectively, and $\mathrm{d} M(u)$ is the differential of the vector $M(u)$ of counting processes, which in the case of "small" $d u$ take values of zero or one for an individual spike train. Let $P_{\mathcal{M}}$ and $P_{\mathcal{N}}$ be the respective mean rates of the processes $\mathcal{M}$ and $\mathcal{N}$, then taking expected values of Eq. (1) taken over realisations of the processes $\mathcal{M}$ and $\mathcal{N}$ gives

$P_{\mathcal{N}}=\mu+\int_{-\infty}^{\infty} K(t-u) P_{\mathcal{M}} \mathrm{d} u$.

The elimination of $\mu$ between Eqs. (1) and (2) gives

$\mu_{\mathcal{N}}(t)-P_{\mathcal{N}}=\int_{-\infty}^{\infty} K(t-u)\left(\mathrm{d} M(u)-P_{\mathcal{M}} \mathrm{d} u\right)$

which describes how departures from the mean rate of the output process $\mathcal{N}$ are related to fluctuations in the input process about its mean rate. In order to determine $K$, Eq. (3) is first multiplied by $\mathrm{d} M(v)-P_{\mathcal{M}} \mathrm{d} v$, and the expectation of the resulting equation then taken with respect to realisations of the processes $\mathcal{M}$ and $\mathcal{N}$ to obtain

$Q_{\mathcal{N M}}(v)=\int_{-\infty}^{\infty} K(v-s) Q_{\mathcal{M M}}(s) \mathrm{d} s$

where $Q_{\mathcal{M M}}(s)$ is the matrix of second order cumulant densities and cross-cumulant densities of $\mathcal{M}$ and $Q_{\mathcal{N M}}(s)$ is the matrix of cross-cumulant densities between the processes $\mathcal{N}$ and $\mathcal{M}$. These densities are defined by

$\operatorname{Cov}\{\mathrm{d} \mathcal{M}(t+v), \mathrm{d} \mathcal{M}(t)\}=\left(P_{\mathcal{M}} \delta(v)+Q_{\mathcal{M M}}(v)\right) \mathrm{d} t \mathrm{~d} v$, $\operatorname{Cov}\{\mathrm{d} \mathcal{N}(t+v), \mathrm{d} \mathcal{M}(t)\}=Q_{\mathcal{N M}}(v) \mathrm{d} t \mathrm{~d} v$,

where Cov denotes covariance and $\delta(t)$ is the Dirac delta function. Equation (4) is a Fourier convolution from which it follows directly that

$$
\begin{aligned}
A(\lambda) & =\mathcal{F}[K(u) ; \lambda] \\
& =\mathcal{F}\left[Q_{\mathcal{N M}}(u) ; \lambda\right]\left(\mathcal{F}\left[Q_{\mathcal{M M}}(u) ; \lambda\right]\right)^{-1}
\end{aligned}
$$

provided the inverse exists. In Eq. (5) $\lambda$ is frequency, $\mathcal{F}[S(u) ; \lambda]$ denotes the Fourier transform of $S(u)$ and $A(\lambda)$ is the transfer function of the system specified in Eq. (1).

\subsection{Error process}

The question of how well the linear model captures the properties of the output process $\mathcal{N}$ can be described by introducing an error process. In the analysis of spike trains, this error process is often formulated in terms of how well the

\footnotetext{
${ }^{1}$ The matrix $K$ may be viewed as the impulse response of the system.
} 
linear model predicts $\mu_{\mathcal{N}}$. The instantaneous error $\varepsilon(t)$ is defined by

$$
\varepsilon(t)=\left(\mu_{\mathcal{N}}(t)-P_{\mathcal{N}}\right)-\int_{-\infty}^{\infty} K(t-v)\left(\mathrm{d} M(v)-P_{\mathcal{M}} \mathrm{d} v\right)
$$

The error process measures the extent to which Eq. (3) is not satisfied, and is assumed to be a stationary process satisfying a mixing condition, i.e. events well separated in time behave approximately independently. When expressed in terms of the error process, the linear model can be restated as

$$
\begin{aligned}
\mu_{\mathcal{N}}(t)= & {\left[P_{\mathcal{N}}-P_{\mathcal{M}} \int_{-\infty}^{\infty} K(v) \mathrm{d} v\right] } \\
& +\int_{-\infty}^{\infty} K(t-v) \mathrm{d} M(v)+\varepsilon(t) .
\end{aligned}
$$

Suppose that the data available for analysis are the pair of vectors $(M(t), N(t))$ where $t \in(0, T]$. The finite Fourier transform applied to the model equation (7) gives

$$
\begin{aligned}
d_{\mu}(\lambda, T)= & 2\left[P_{\mathcal{N}}-P_{\mathcal{M}} \int_{-\infty}^{\infty} K(v) \mathrm{d} v\right] \sin (\lambda T / 2) \mathrm{e}^{-\mathrm{i} \lambda T / 2} \\
& +\int_{0}^{T} \mathrm{e}^{-\mathrm{i} \lambda t}\left[\int_{-\infty}^{\infty} K(t-v) \mathrm{d} M(v)\right] \mathrm{d} t \\
& +d_{\varepsilon}(\lambda, T)
\end{aligned}
$$

where $d_{\mu}(\lambda, T)$ is the finite Fourier transform of $\mu_{\mathcal{N}}(t)$ and $d_{\varepsilon}(\lambda, T)$ is the finite Fourier transform of the error process $\varepsilon(t)$ at frequency $\lambda$. Specifically,

$$
\begin{aligned}
d_{\mu}(\lambda, T) & =\int_{0}^{T} \mathrm{e}^{-\mathrm{i} \lambda t} \mu_{\mathcal{N}}(t) \mathrm{d} t \quad \text { while } \\
d_{\mathcal{N}}(\lambda, T) & =\int_{0}^{T} \mathrm{e}^{-\mathrm{i} \lambda t} \mathrm{~d} N(t) .
\end{aligned}
$$

In the computations one chooses $\lambda=\lambda_{n}=2 \pi n / T$ where $n=1,2, \ldots, T-1$ which results in the elimination of the first term on the right-hand side of Eq. (8) to obtain

$$
\begin{aligned}
d_{\mathcal{N}}\left(\lambda_{n}, T\right)= & \int_{0}^{T} \mathrm{e}^{-\mathrm{i} \lambda_{n} t}\left[\int_{-\infty}^{\infty} K(t-v) \mathrm{d} M(v)\right] \mathrm{d} t \\
& +d_{\varepsilon}\left(\lambda_{n}, T\right) .
\end{aligned}
$$

Changing the order of integration in the integral on the right hand side of this equation gives

$$
\begin{aligned}
d_{\mathcal{N}}\left(\lambda_{n}, T\right)= & \int_{-\infty}^{\infty} \mathrm{e}^{-\mathrm{i} \lambda_{n} v} K(v)\left[\int_{-v}^{T-v} \mathrm{e}^{-\mathrm{i} \lambda_{n} u} \mathrm{~d} M(u)\right] \mathrm{d} v \\
& +d_{\varepsilon}\left(\lambda_{n}, T\right) .
\end{aligned}
$$

Since $\mathcal{M}$ is assumed to be a stationary process, and $\mathrm{e}^{-\mathrm{i} \lambda_{n} u}$ has period $T$ in $u$, then for large $T$

$$
\int_{-v}^{T-v} \mathrm{e}^{-\mathrm{i} \lambda_{n} u} \mathrm{~d} M(u) \approx \int_{0}^{T} \mathrm{e}^{-\mathrm{i} \lambda_{n} u} \mathrm{~d} M(u)=d_{\mathcal{M}}\left(\lambda_{n}, T\right)
$$

where the approximation arises as a consequence of the properties of $K(u)$, the mixing properties and sampling variability of the process $\mathcal{M}$ over the interval of duration $T$. With this approximation in place, Eq. (10) becomes

$d_{\mathcal{N}}\left(\lambda_{n}, T\right) \approx A(\lambda) d_{\mathcal{M}}\left(\lambda_{n}, T\right)+d_{\varepsilon}\left(\lambda_{n}, T\right)$,

(for $\lambda_{n}$ near $\lambda$ ),

where $A(\lambda)$, assumed continuous at $\lambda$, is the Fourier transform of $K(u)$. To facilitate the development of subsequent analysis, it is convenient to introduce the notation $\xi(\lambda)=$ $d_{\mathcal{N}}(\lambda, T), \eta(\lambda)=d_{\mathcal{M}}(\lambda, T)$ and $e(\lambda)=d_{\varepsilon}(\lambda, T)$ so that Eq. (11) has the simplified representation

$\xi\left(\lambda_{n}\right) \approx A(\lambda) \eta\left(\lambda_{n}\right)+e\left(\lambda_{n}\right), \quad\left(\right.$ for $\lambda_{n}$ near $\left.\lambda\right)$.

If $Z(t)$ is a stationary vector process satisfying a mixing condition, then each Fourier coefficient $d_{Z}\left(\lambda_{n}, T\right)$ behaves asymptotically as an independently distributed complexnormal deviate with mean value zero and covariance matrix $2 \pi T f_{Z Z}(\lambda)$ (Brillinger 1983). Consequently, the Fourier coefficients $d_{\varepsilon}\left(\lambda_{n}, T\right)$ of $\varepsilon(t)$ behave asymptotically as independently distributed complex-normal deviates ${ }^{2}$ with mean value zero and covariance matrix $2 \pi T f_{\varepsilon \varepsilon}(\lambda)$. This property of the error process suggests that Eq. (12) can be interpreted as a standard linear model in which the expected value of $\xi(\lambda)$, estimated with respect to realisations of $\xi(\lambda)$ formed by partitioning observations into samples of duration $T$, is approximated by $A(\lambda) \eta(\lambda)$. It is to be noted that if the matrix $A(\lambda)$ is chosen to minimise the matrix

$$
\begin{aligned}
& \Phi(A) \\
& =\frac{1}{2 \pi T} \mathbb{E}\left[(\xi(\lambda)-A(\lambda) \eta(\lambda))(\xi(\lambda)-A(\lambda) \eta(\lambda))^{\mathrm{H}}\right],
\end{aligned}
$$

\footnotetext{
2 A random variable is said to be a multivariate complex-normal deviate if its components are complex valued and its probability density function is multivariate Gaussian with a Hermitian covariance of a particular form (see Brillinger 1975b, page 89, equations (4.2.4) and (4.2.5), and the references therein).
} 
then $A(\lambda)=\mathbb{E}\left[\xi(\lambda) \eta(\lambda)^{\mathrm{H}}\right] \mathbb{E}\left[\eta(\lambda) \eta(\lambda)^{\mathrm{H}}\right]^{-1}$. The value of $\Phi(A)$ at its minimum is $\mathbb{E}\left[e(\lambda) e(\lambda)^{\mathrm{H}}\right] / 2 \pi T$ with $e(\lambda)=$ $\xi(\lambda)-A(\lambda) \eta(\lambda)$ and for large $T$ approximates the spectral density matrix of the error process. Note also that because Fourier coefficients are complex-valued functions of frequency, the usual matrix operation of transposition, namely $\eta^{\mathrm{T}}$ in the case of a real-valued vector $\eta(\lambda)$, must be replaced by the Hermitian operation of taking the complex conjugate of the transposed matrix, namely $\eta^{\mathrm{H}}$ in the case of the complex-valued vector $\eta(\lambda)$.

The spectral density matrices $f_{\mathcal{N} \mathcal{M}}(\lambda)$ and $f_{\mathcal{M M}}(\lambda)$ are defined by

$$
\begin{aligned}
f_{\mathcal{M M}}(\lambda) & \approx \frac{1}{2 \pi T} \mathbb{E}\left[\eta(\lambda) \eta^{\mathrm{H}}(\lambda)\right], \\
f_{\mathcal{N M}}(\lambda) & \approx \frac{1}{2 \pi T} \mathbb{E}\left[\xi(\lambda) \eta^{\mathrm{H}}(\lambda)\right]
\end{aligned}
$$

for large $T$. The corresponding Fourier coefficients of the error process and the associated spectral density matrix at this minimum are, respectively,

$e(\lambda)=\xi(\lambda)-A(\lambda) \eta(\lambda)$.

Taking $K$ in (6) to be the inverse Fourier transform of $f_{\mathcal{N M}}(\lambda) f_{\mathcal{M M}}^{-1}(\lambda)$ one obtains

$f_{\varepsilon \varepsilon}(\lambda)=f_{\mathcal{N N}}(\lambda)-f_{\mathcal{N} \mathcal{M}}(\lambda) f_{\mathcal{M} \mathcal{M}}^{-1}(\lambda) f_{\mathcal{N} \mathcal{M}}^{\mathrm{H}}(\lambda)$.

Moreover $\mathrm{E}\left[e(\lambda) \eta(\lambda)^{\mathrm{H}}\right]=0$ and the error process $\varepsilon(t)$ and input process $\mathcal{M}$ are uncorrelated at each frequency $\lambda$. The right-hand side of Eq. (16) is the spectral density matrix of the error process, that is, it is the component of the spectral density matrix of the output process that cannot be predicted from the input process based on a linear model. For this reason $f_{\varepsilon \varepsilon}(\lambda)$ is called the partial spectrum of $\mathcal{N}$ having removed the effect of $\mathcal{M}$ in a linear time invariant manner and is denoted by $f_{\mathcal{N N} \mid \mathcal{M}}$. Further details may be found in Brillinger (1975b) where tests of hypotheses are also given. In practice the quantities in expression (16) are replaced by their estimates so that

$\widehat{f}_{\varepsilon \mathcal{E}}\left(\lambda_{n}\right)=\widehat{f}_{\mathcal{N} \mathcal{N}}\left(\lambda_{n}\right)-\widehat{f}_{\mathcal{N} \mathcal{M}}\left(\lambda_{n}\right) \widehat{f}_{\mathcal{M} \mathcal{M}}^{-1}\left(\lambda_{n}\right) \widehat{f}_{\mathcal{N} \mathcal{M}}^{\mathrm{H}}\left(\lambda_{n}\right)$.

\subsection{Coherency}

In common practice the coherency between a single input process $\mathcal{M}$ and a single output process $\mathcal{N}$ is defined by

$R_{\mathcal{N M}}=\frac{f_{\mathcal{N M}}}{\sqrt{f_{\mathcal{N N}}} \sqrt{f_{\mathcal{M M}}}}$,

and the spectrum of the error incurred in using the linear model to predict the output process $\mathcal{N}$ from the input process $\mathcal{M}$ is

$f_{\varepsilon \varepsilon}=f_{\mathcal{N N}}\left(1-\left|R_{\mathcal{N} \mathcal{M}}\right|^{2}\right)$ where $\left|R_{\mathcal{N} M}\right|^{2}$ is the coherence between the processes $\mathcal{M}$ and $\mathcal{N}$. By construction, the coherence $\left|R_{\mathcal{N} \mathcal{M}}\right|^{2}$ takes a value between zero and one where a coherence of zero means that the linear model has no power to explain the output process $\mathcal{N}$ from the input process $\mathcal{M}$.

Given vector-valued point processes $\mathcal{N}$ and $\mathcal{M}$, the scalar coherency (18) in the single-input single-output case is replaced by the coherency matrix $R_{\mathcal{N M}}(\lambda)$ defined by

$f_{\mathcal{N} \mathcal{M}}(\lambda)=D_{\mathcal{N}}(\lambda) R_{\mathcal{N} \mathcal{M}}(\lambda) D_{\mathcal{M}}(\lambda)$,

where $D_{\mathcal{N}}$ and $D_{\mathcal{M}}$ are the diagonal matrices formed from the square roots of the diagonal entries of the respective spectral density matrices $f_{\mathcal{N N}}$ and $f_{\mathcal{M M}}$. The spectrum of the error process corresponding to Eq. (19) in the single-input single-output case is replaced by

$f_{\varepsilon \varepsilon}=D_{\mathcal{N}}\left(R_{\mathcal{N N}}-R_{\mathcal{N} \mathcal{M}} R_{\mathcal{M} \mathcal{M}}^{-1} R_{\eta \xi}\right) D_{\mathcal{N}}^{\mathrm{H}}$

in which $R_{\mathcal{N} \mathcal{N}}$ and $R_{\mathcal{M M}}$ are Hermitian matrices and $R_{\mathcal{M M}}^{-1}$ is assumed to exist. A fundamental property of $R_{\mathcal{N N}}$ and $R_{\mathcal{M M}}$ is that they have unique Choleski factorisations $R_{\mathcal{N N}}=L_{\mathcal{N}} L_{\mathcal{N}}^{\mathrm{H}}$ and $R_{\mathcal{M M}}=L_{\mathcal{M}} L_{\mathcal{M}}^{\mathrm{H}}$ in which $L_{\mathcal{N}}$ and $L_{\mathcal{M}}$ are lower triangular matrices with positive entries in their main diagonals. With the appropriate substitutions in expression (21), the spectral density matrix of the error process becomes

$f_{\varepsilon \varepsilon}=\left(D_{\mathcal{N}} L_{\mathcal{N}}\right)\left(I-R R^{\mathrm{H}}\right)\left(D_{\mathcal{N}} L_{\mathcal{N}}\right)^{\mathrm{H}}$,

where $R=L_{\mathcal{N}}^{-1} R_{\mathcal{N M}}\left(L_{\mathcal{M}}^{-1}\right)^{\mathrm{H}}$. In the case of a single-input single-output model, expression (19) is recovered from expression (22). The matrix $\left(I-R R^{\mathrm{H}}\right)$ in expression (22) describes the fraction of the output spectrum that is not predicted from the linear model. By construction, $R R^{\mathrm{H}}$ is a positive definite Hermitian matrix. Since by definition $f_{\varepsilon \varepsilon}$ is a complex-valued spectral density matrix, then $Z^{\mathrm{H}} f_{\varepsilon \varepsilon} Z \geq 0$ for column vectors $Z$ and so it follows directly from expression (22) that

$Y^{\mathrm{H}} Y \geq Y^{\mathrm{H}}\left(R R^{\mathrm{H}}\right) Y$

where $Y=D_{\mathcal{N}} L_{\mathcal{N}}^{\mathrm{H}} Z$. Consequently the eigenvalues of $R R^{\mathrm{H}}$ lie in the interval $[0,1]$ which in turn suggests that $R$ may play the role of a generalised coherency and that the eigenvalues of $R R^{\mathrm{H}}$ may be called coherences.

However, in order to call $R$ a coherency it is necessary to identify underlying processes, say $\mathcal{X}$ and $\mathcal{Z}$, for which $R$ is the coherency. Consider the process with stationary finite Fourier transform $d_{\mathcal{X}}(\lambda)=L_{\mathcal{N}}^{-1} D_{\mathcal{N}}^{-1} \xi(\lambda)$ and $d_{\mathcal{Z}}(\lambda)=$ $L_{\mathcal{M}}^{-1} D_{\mathcal{M}}^{-1} \eta(\lambda)$ which are constructed from linear combinations of the Fourier coefficients $\xi(\lambda)$ and $\eta(\lambda)$ of the processes $\mathcal{N}$ and $\mathcal{M}$. It is direct to show that the spectral matrices of $\mathcal{X}$ and $\mathcal{Z}$ are $I$ which means that the corresponding frequency components of $\mathcal{X}$ and of $\mathcal{Z}$ are uncorrelated random 
variables with unit variance. The cross-spectrum between $\mathcal{X}$ and $\mathcal{Z}$ is $R_{\mathcal{X Z}}=L_{\mathcal{N}}^{-1} R_{\mathcal{N} \mathcal{M}}\left(L_{\mathcal{M}}^{-1}\right)^{\mathrm{H}}=R$ since the spectral matrices of the processes $\mathcal{X}$ and $\mathcal{Z}$ are both identity matrices. Thus $R$ is a coherency matrix and the eigenvalues of $R R^{\mathrm{H}}$ are coherences. Such coherences are called canonical coherences (Brillinger 1975b, Section 10.3). Specifically, if $R R^{\mathrm{H}}=I$ then the linear model predicts $\mathcal{N}$ from $\mathcal{M}$ with no error, whereas if $R R^{\mathrm{H}}=0$ then the linear model has no power to predict $\mathcal{N}$ from $\mathcal{M}$.

\subsection{Spectral decomposition of the error process}

For the purpose of investigating the behaviour of a network, the spectral density matrix of the error process $f_{\varepsilon \varepsilon}$ can be decomposed into a series of terms each of which expresses a different contribution to the error process. These contributions can be thought of as representing how different patterns of connectivity contribute to the error within the network. This decomposition of the error process is obtained by recognising that there is a unitary matrix $U$, i.e. a matrix satisfying $U^{\mathrm{H}} U=I$, with the property that

$R R^{\mathrm{H}}=U D_{\psi} U^{\mathrm{H}}=\sum_{k=1}^{J} \psi_{k} U_{k} U_{k}^{\mathrm{H}}$,

where $J$ is the dimension of $R R^{\mathrm{H}}, D_{\psi}$ is the diagonal matrix of the eigenvalues $\psi_{1}, \ldots, \psi_{J}$ of $R R^{\mathrm{H}}$ and $U_{k}$ is the $k$ th column of $U$, the unitary matrix diagonalising $R R^{\mathrm{H}}$. This representation of $R R^{\mathrm{H}}$ allows the error process to be re-expressed in the form

$f_{\varepsilon \varepsilon}=D_{\mathcal{N}} L_{\mathcal{N}} U\left(I-D_{\psi}\right) U^{\mathrm{H}} L_{\mathcal{N}}^{\mathrm{H}} D_{\mathcal{N}}$.

Let $C_{k}$ be the $k$ th column of $D_{\mathcal{N}} L_{\mathcal{N}} U$, then $C_{k}=D_{\mathcal{N}} L_{\mathcal{N}} U_{k}$ and expression (24) becomes

$f_{\varepsilon \varepsilon}=\sum_{k=1}^{J}\left(1-\psi_{k}\right) C_{k} C_{k}^{\mathrm{H}}$

An interpretation of expression (25) is obtained by observing first that $C=\left[C_{1} \cdots C_{J}\right]$ satisfies

$f_{\mathcal{N} \mathcal{N}}=C C^{\mathrm{H}}=\sum_{k=1}^{J} C_{k} C_{k}^{\mathrm{H}}$.

Equation (26) decomposes the spectrum of $\mathcal{N}$ into a sum of spectral density matrices, and therefore expression (25) can be interpreted as a weighted sum of the elements of this decomposition in which each weighting factor is a coherence. Of course, the structure of this decomposition and the coherence will depend on the configuration of the system. The application of expression (25) will be illustrated both analytically and numerically by an analysis of the results of an experiment in which data from two input point processes and two output point processes are available.

\subsection{Analytical example}

The application of expression (25) to the case of two output processes with either one input process or two input processes will be treated analytically and used to illustrate how the spectral density of the error process is decomposed. For representational convenience, the dependence of all expressions on $\lambda$ will be suppressed and the input and output bivariate processes will be expressed in terms of their component processes by

$\mathcal{M}=\left[\begin{array}{l}\mathcal{A} \\ \mathcal{B}\end{array}\right], \quad \mathcal{N}=\left[\begin{array}{l}\mathcal{C} \\ \mathcal{D}\end{array}\right]$

If only one input process, say $\mathcal{A}$, is considered then it is straightforward to show that

$$
\begin{aligned}
& R R^{\mathrm{H}} \\
& \quad=\left[\begin{array}{cc}
\left|R_{\mathcal{C} \mathcal{A}}\right|^{2} & R_{\mathcal{C} \mathcal{A}} R_{\mathcal{A D} \mid \mathcal{C}}\left(1-\left|R_{\mathcal{C A}}\right|^{2}\right)^{1 / 2} \\
R_{\mathcal{A C}} R_{\mathcal{D} \mathcal{A} \mid \mathcal{C}}\left(1-\left|R_{\mathcal{C} \mathcal{A}}\right|^{2}\right)^{1 / 2} & \left|R_{\mathcal{D} \mathcal{A} \mid \mathcal{C}}\right|^{2}\left(1-\left|R_{\mathcal{C} \mathcal{A}}\right|^{2}\right)
\end{array}\right],
\end{aligned}
$$

where $R_{\mathcal{A B} \mid \mathcal{C}}$ denotes the partial coherency of the processes $\mathcal{A}$ and $\mathcal{B}$ conditioned on the process $\mathcal{C}$ and is defined in terms of the ordinary coherencies $R_{\mathcal{A B}}, R_{\mathcal{A C}}$ and $R_{\mathcal{C B}}$ by the formula

$R_{\mathcal{A B} \mid \mathcal{C}}=\frac{R_{\mathcal{A B}}-R_{\mathcal{A C}} R_{\mathcal{C B}}}{\sqrt{1-\left|R_{\mathcal{A C}}\right|^{2}} \sqrt{1-\left|R_{\mathcal{C B}}\right|^{2}}}$.

Moreover, the matrix $R R^{\mathrm{H}}$ has eigenvalues

$\psi_{1}=0, \quad \psi_{2}=\left|R_{\mathcal{C} \mathcal{A}}\right|^{2}+\left|R_{\mathcal{D} \mathcal{A} \mid \mathcal{C}}\right|^{2}\left(1-\left|R_{\mathcal{C} \mathcal{A}}\right|^{2}\right)$

and corresponding eigenvectors

$U_{1}=\frac{1}{\sqrt{\psi_{2}}}\left[\begin{array}{c}R_{\mathcal{A D} \mid \mathcal{C}}\left(1-\left|R_{\mathcal{C} \mathcal{A}}\right|^{2}\right)^{1 / 2} \\ -R_{\mathcal{A C}}\end{array}\right]$,
$U_{2}=\frac{1}{\sqrt{\psi_{2}}}\left[\begin{array}{c}R_{\mathcal{C} \mathcal{A}} \\ R_{\mathcal{D} \mathcal{A} \mid \mathcal{C}}\left(1-\left|R_{\mathcal{C} \mathcal{A}}\right|^{2}\right)^{1 / 2}\end{array}\right]$

where it is assumed that $\psi_{2} \neq 0$. Expressions (28), (30) and (31) make explicit how the interactions between the output processes and a single input process contribute to the structure of the error process. To appreciate why this is the case, expression (26) decomposes the output process into components parts each of which is scaled by a coherence in Eq. (25) to give the error spectrum. The scaling factor is an eigenvalue of $R R^{\mathrm{H}}$, which expression (30) shows will incorporate coherences and products of coherences between the input and output processes. If two input processes $\mathcal{A}$ and $\mathcal{B}$ are present, then a more complicated calculation leads to the coherency matrix 


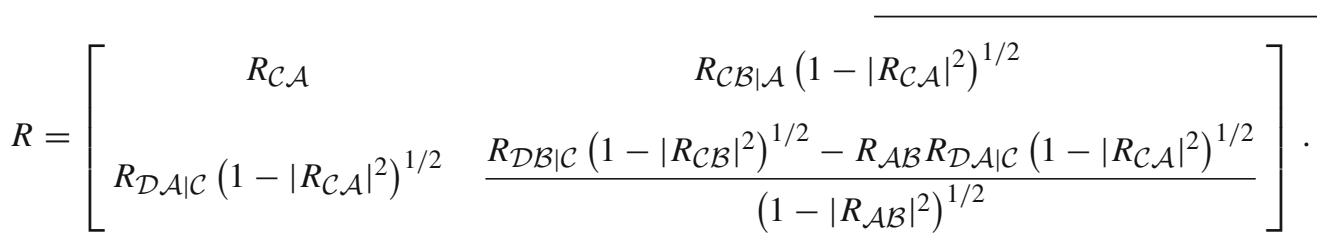

Since in our example the input processes $\mathcal{A}$ and $\mathcal{B}$ are generated to be statistically independent, then $R_{\mathcal{A B}}=0$ and the coherency matrix $R$ takes the simplified form

$R=\left[\begin{array}{cc}R_{\mathcal{C} \mathcal{A}} & R_{\mathcal{C B}} \\ R_{\mathcal{D} \mathcal{A} \mid \mathcal{C}}\left(1-\left|R_{\mathcal{C A}}\right|^{2}\right)^{1 / 2} & R_{\mathcal{D B} \mid \mathcal{C}}\left(1-\left|R_{\mathcal{C B}}\right|^{2}\right)^{1 / 2}\end{array}\right]$

with associated coherence matrix

$$
\begin{aligned}
& R R^{\mathrm{H}}=\left[\begin{array}{ll}
R_{11} & R_{12} \\
R_{21} & R_{22}
\end{array}\right], \\
& R_{11}=\left|R_{\mathcal{C} \mathcal{A}}\right|^{2}+\left|R_{\mathcal{B C}}\right|^{2}, \\
& R_{12}=R_{\mathcal{C A}} R_{\mathcal{A D} \mid \mathcal{C}} \sqrt{1-\left|R_{\mathcal{C A}}\right|^{2}}+R_{\mathcal{C B}} R_{\mathcal{B D} \mid \mathcal{C}} \sqrt{1-\left|R_{\mathcal{C B}}\right|^{2}}, \\
& R_{21}=R_{\mathcal{A C}} R_{\mathcal{D} \mathcal{A} \mid \mathcal{C}} \sqrt{1-\left|R_{\mathcal{C} \mathcal{A}}\right|^{2}}+R_{\mathcal{B C}} R_{\mathcal{D B} \mid \mathcal{C}} \sqrt{1-\left|R_{\mathcal{C B}}\right|^{2}}, \\
& R_{22}=\left|R_{\mathcal{D} \mathcal{A} \mid \mathcal{C}}\right|^{2}\left(1-\left|R_{\mathcal{C} \mathcal{A}}\right|^{2}\right)+\left|R_{\mathcal{D B} \mid \mathcal{C}}\right|^{2}\left(1-\left|R_{\mathcal{C B}}\right|^{2}\right) \text {. }
\end{aligned}
$$

In the case of two input processes and two output processes expression (32) makes explicit how the input and output processes interact without an assumption as to whether or not the input processes are independent. Expression (34) is the coherence matrix of input and output processes in the special case in which the two input processes have coherence zero. These interactions will be examined in detail in Sect. 4.

\subsection{Numerical example}

An immediate difficulty in assessing the error in the behaviour of a model with multiple input processes and multiple output processes (not present in problems involving a single input process and single output process) is the question of how to measure the size of matrices arising in the analysis of these systems. A standard procedure used in numerical analysis is to measure the size of a matrix by means of a norm, which in this article is chosen to be the magnitude of the eigenvalue of largest modulus (Hermitian norm). Interestingly, the transfer function $A(\lambda)=\mathbb{E}\left[\xi(\lambda) \eta(\lambda)^{\mathrm{H}}\right]$ $\mathbb{E}\left[\eta(\lambda) \eta(\lambda)^{\mathrm{H}}\right]^{-1}$ minimises the Hermitian norm of the matrix $\Phi(A)$ in expression (13).

If the linear model cannot predict features of the output process given the input process, then the spectral density matrix of the error process will be identical to that of the output process. Thus the norm of the spectral density matrix of the output process provides reference values against which the adequacy of the linear model can be assessed. Figure 2 gives an example of the use of this approach in assessing the suitability of the linear model to explain the relationship between the input and output for the muscle spindle experiment.

The upper and lower panels of Fig. 2 represent respectively the analysis of the behaviour of the muscle spindle in the absence and in the presence of imposed length changes. In this figure the adequacy of the linear model is tested when only one input process is taken into account (dotted line) although both inputs are active, and when both input processes are taken into account (dashed line) in the estimation of the Hermitian norm of the error process. The upper left hand panel of Fig. 2 suggests that the linear model is not adequate at frequencies in excess of $20 \mathrm{~Hz}$, but that it may be adequate at frequencies below $20 \mathrm{~Hz}$ in the absence of an imposed length change when both input processes are taken into account (see upper right hand panel which expands the lower left hand corner of the upper left hand panel). The lower panels of Fig. 2 illustrate that the linear model is not adequate at any frequency in the presence of a dynamic length change.

The behaviour of the norm of the error process in Fig. 2 can be appreciated in terms of the behaviour of the eigenvalues illustrated in Fig. 3. Recall that one eigenvalue $\left(\psi_{1}\right)$ is zero when examining the panels of the left hand column of Fig. 3. In the absence of an imposed length change, Fig. 3 (upper panels) shows that when taking into account a second input (upper right panel), at frequencies below $25 \mathrm{~Hz}$ $\psi_{1}$ becomes nonzero and the second eigenvalue $\psi_{2}$ increases its value with a corresponding reduction in the size of the error process (Fig. 2). On the other hand, in the presence of an imposed length change (lower panels), $\psi_{2}$ (larger eigenvalue) shows no appreciable change when the second input is taken into account, although the presence of the second input causes $\psi_{1}$ to become non-zero. Moreover, the presence of an imposed length change when both input processes are taken into account leads to an appreciable reduction in the value of $\psi_{2}$, leaving $\psi_{1}$ largely unaffected. It suggests, therefore, that the norm of the error process is principally controlled by the value of $\psi_{2}$.

The previous discussion has focussed on the relationship between the eigenvalues of the generalised coherence matrix 
Fig. 2 The Hermitian norm of the estimated spectral density of the output process (solid line) in the absence (upper panels) and presence (lower panels) of an imposed dynamic length change. In each panel dotted lines denote the estimated Hermitian norm of the error process when both $\gamma$ inputs are active but only the $\gamma_{1}$ input is used in the estimation of this norm, whereas dashed lines denote the Hermitian norm of the error process taking into account both of the $\gamma$ inputs in estimating this norm. Right hand panels are enlarged versions of the bottom left hand corner of the left hand panels. Note that when the estimated Hermitian norm of the error process does not differ from that of the output process then the linear model is an inadequate representation of the behaviour of the system
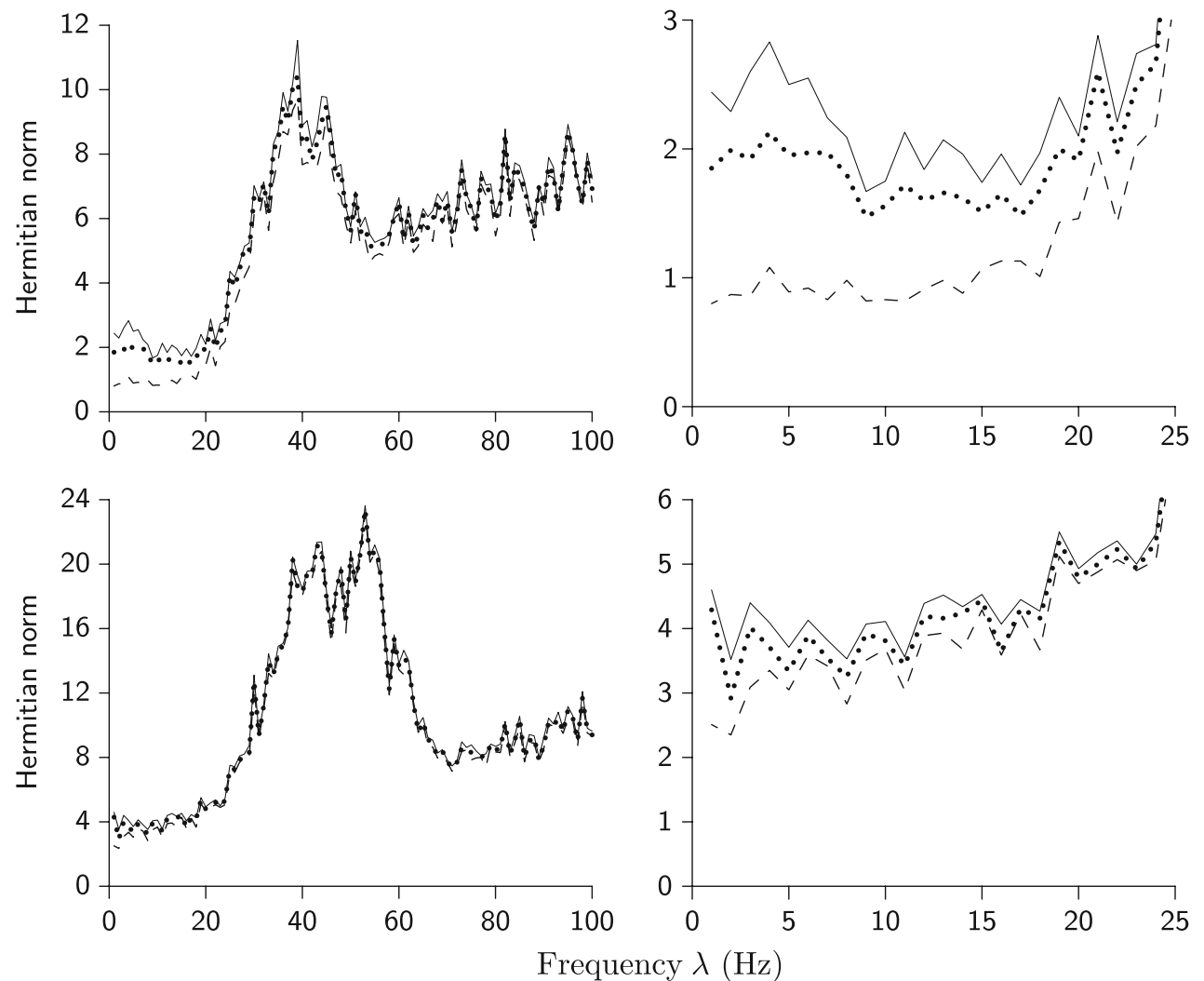

Frequency $\lambda(\mathrm{Hz})$

Fig. 3 When both $\gamma$ inputs are active and the muscle spindle is held at a fixed length, the upper panels compare the estimated nonzero eigenvalue $\left(\psi_{2}\right)$ of $R R^{\mathrm{H}}$ taking account of the $\gamma_{1}$ input alone (left panel) and the eigenvalues $\psi_{1}$ (dotted lines) and $\psi_{2}$ (solid lines) taking account of both $\gamma_{1}$ and $\gamma_{2}$ inputs (right panel). The lower panels show the same eigenvalues for the same combinations of inputs but with imposed length changes. Note that the eigenvalues $\psi_{1}$ and $\psi_{2}$ control the norm of the error process

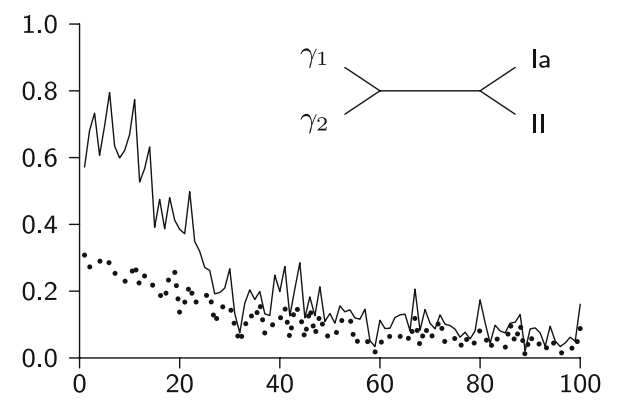

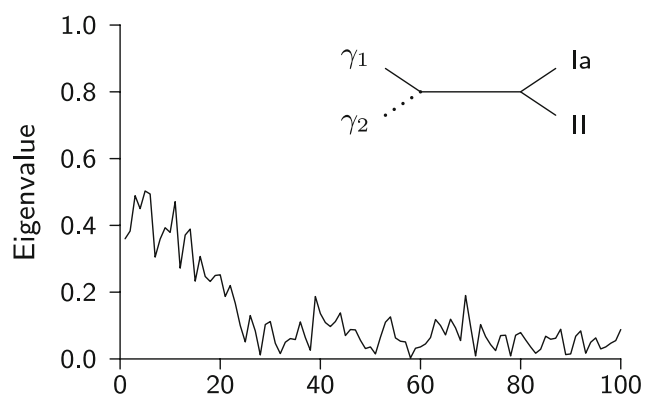

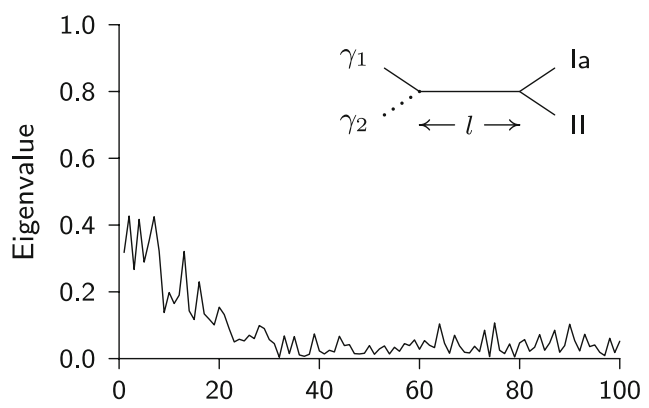

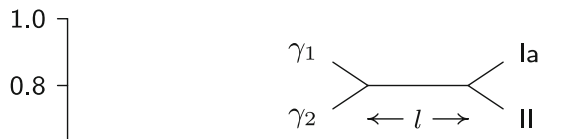

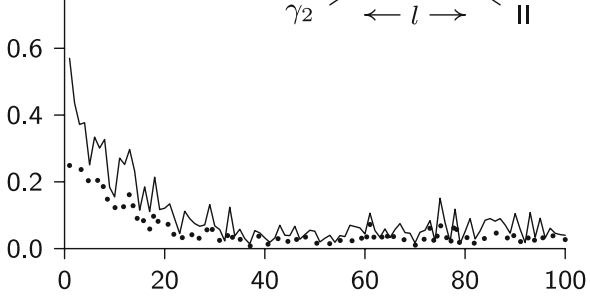

Frequency $\lambda(\mathrm{Hz})$

and the behaviour of the norm of the error process. The behaviour of the eigenvalues themselves can be appreciated in terms of the coherence between the separate input and output processes. Figure 4 (left column) illustrates how the coherence between one input and the response of the second- ary ending (upper left panel) is influenced by the presence of the second input (middle left panel) and by the presence of the second input and an imposed length change (lower left panel). Although the presence of a second input and an imposed length change reduces the coherence between the 
Fig. 4 The left column shows the estimated coherence between the $\gamma_{1}$ input and the II output when the $\gamma_{2}$ input is absent (upper left), in the presence of the $\gamma_{2}$ input (middle left) and in the presence of the $\gamma_{2}$ input with imposed length changes (lower left). The right column shows the coherence between the $\gamma_{1}$ input and the Ia output in the absence of $\gamma_{2}$ (upper right), in the presence of $\gamma_{2}$ input (middle right) and in the presence of the $\gamma_{2}$ input with imposed length changes (lower right). Solid lines in the insert of each panel represent the processes between which the coherence is estimated, whereas dotted lines indicate other processes present and $\leftarrow l \rightarrow$ indicates the presence of an imposed dynamic length change. The horizontal dashed line in each panel represents the upper level of a marginal $95 \%$ confidence interval based on the hypothesis that both processes are independent
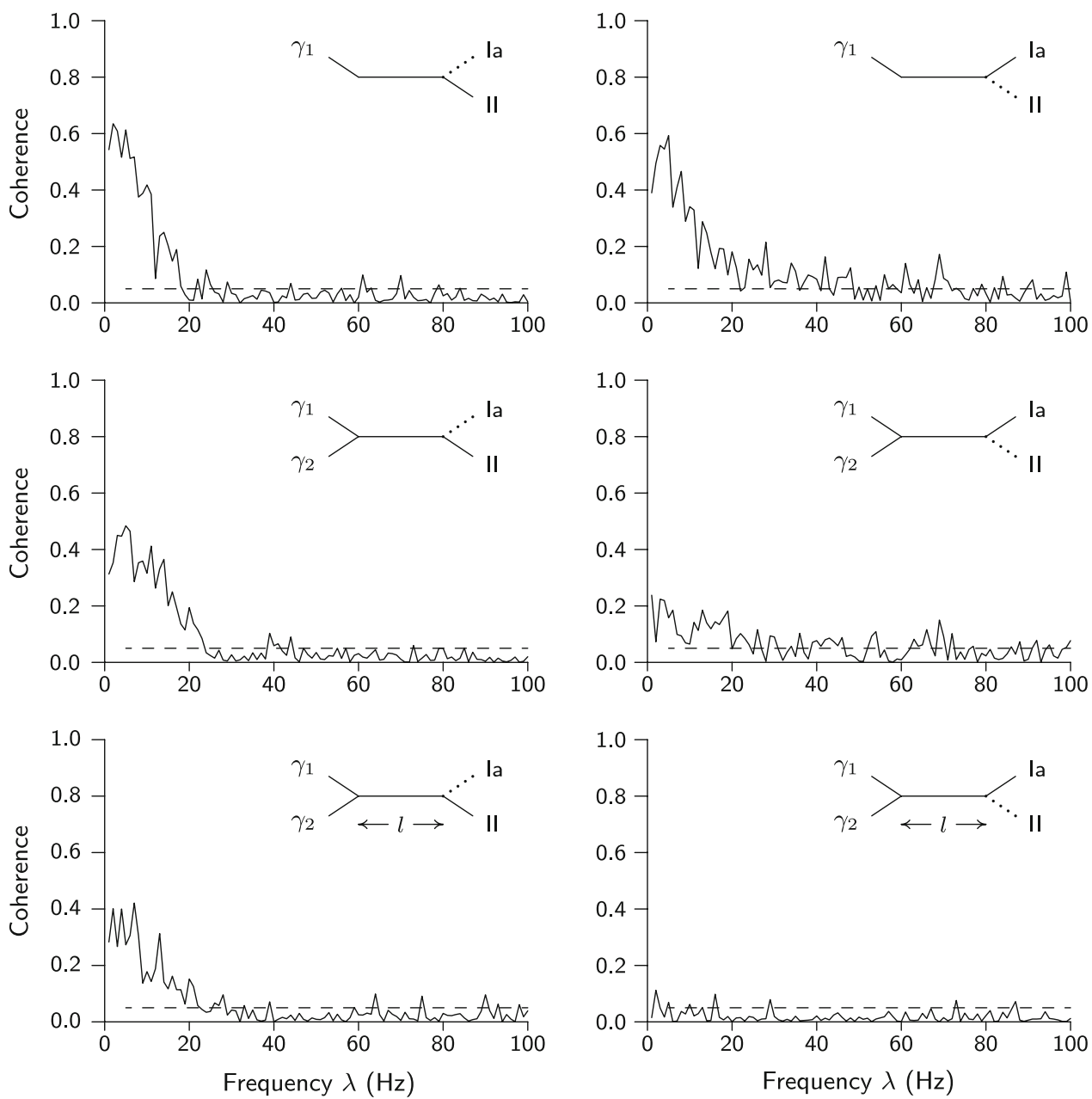

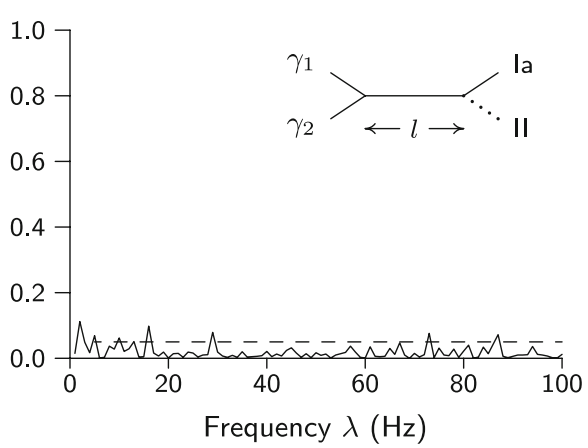

single input and the response of the secondary ending, the coherence between these two processes nevertheless remains significant at frequencies below $20 \mathrm{~Hz}$. The corresponding panels on the right hand column of Fig. 4 indicate that the coherence between the single input and the response of the primary ending is reduced in the presence of the second input, and is not significant in the presence of the second input and imposed length changes.

\section{A time domain approach}

The time domain approach to understanding input-output dependencies for multivariate spike train processes developed in this section involves an extension of the rate function $\mu_{\mathcal{N}}$ given in expression (1) by making use of the complete intensity function defined by

$$
\begin{aligned}
& \mu_{\mathcal{N}}(t \mid \mathcal{M}(u), \mathcal{N}(u), u \leq t) \\
& =\frac{1}{h} \operatorname{Prob}\{\mathcal{N} \text { spike in }(t, t+h] \mid \mathcal{M}(u), \mathcal{N}(u), u \leq t\}
\end{aligned}
$$

for $h$ small. This function completely describes the stochastic properties of many temporal point processes. A distinction of this approach is that the motivating model is nonlinear (Lindsay et al. 2001), and uses the spike generating mechanism and recovery process described in Brillinger and Segundo (1979) extended to the case of several point process inputs. In this model the input process $\mathcal{M}$ and the membrane potential $U(t)$ resulting from this input are related through the equation

$U(t)=\int_{0}^{b} a(u) \mathrm{d} M(t-u)$

where $a(u)$ is called a summation function, $b$ is the duration of the interval to the preceding output spike and $M$ is the step unit function corresponding to the times of input spikes. The function $a(u)$ in Eq. (36) plays the role of $K(u)$ in Eq. (1), and estimates of its Fourier transform might be compared with estimates of $A(\lambda)$ in Eq. (5). An output spike is generated from the model (36) whenever $U(t) \geq \theta(t)=\theta+\epsilon(t)$ where $\theta$ is a parameter of the model and $\epsilon(t)$ is a noise process. Upon spike initiation, the function $U(t)$ is reset to zero. 
In the computational work presented in this section, the spike trains $\mathcal{M}(t)$ and $\mathcal{N}(t)$ are represented by the time series $M_{t}$ and $N_{t}$ with $M_{t}=1\left(N_{t}=1\right)$ if a spike occurred in the interval $(t-1, t]$ and zero otherwise. In addition, the functions $U(t)$ and $\theta(t)$ are replaced by $U_{t}$ and $\theta_{t}$ respectively and $\epsilon(t)$ by $\epsilon_{t}$ to highlight its discreteness, with $t= \pm 1, \pm 2, \ldots$. The summation function $a(u)$ will be approximated by $a_{u}$ with $u=1,2, \ldots$ so that expression (36) now takes the form

$U_{t}=\sum_{u=0}^{b} a_{u} M_{t-u}$

The values, $\epsilon_{t}$, of the noise process values will be assumed to be independent and identically distributed with distribution function $P(e)=\operatorname{Prob}\left\{\epsilon_{t} \leq e\right\}$. The complete intensity function is then approximated by

$\operatorname{Prob}\left\{N_{t}=1 \mid U_{t}\right\}=\operatorname{Prob}\left\{U_{t} \geq \theta+\epsilon_{t}\right\}=P\left(U_{t}-\theta\right)$.

In the subsequent analyses $\epsilon_{t}$ will have a logistic distribution for ease of computations, although it could have been assumed to be normally distributed. This model is referred to as a Logit model. The principal statistical computer packages ${ }^{3}$ have functions for fitting this model with those functions providing estimates of the parameters and their uncertainties. These models are particular cases of the generalized linear model (McCullagh and Nelder 1989). More generally, $P$ itself may be estimated if desired (Brillinger 1992).

When the data, $\left(M_{t}, N_{t}\right), t=0, \ldots, T-1$, of the corresponding input and output series are available, estimation of the summation function $a$ can proceed by maximizing the likelihood

$$
\prod_{t=0}^{T-1} P\left(U_{t}-\theta\right)^{N_{t}}\left[1-P\left(U_{t}-\theta\right)\right]^{1-N_{t}}
$$

as a function of $a_{0}, \ldots, a_{b}$. To obtain smoothed estimates of the values of the function $a(u)$ at $u=0,1, \ldots, b$, regularized maximum likelihood estimates are employed (Vapnik 1982). The procedure is to add a penalty term, $R$, and regularization parameter, $\gamma$, to the log of the likelihood function (39) to give the penalized likelihood function

$$
\begin{aligned}
& \sum_{t}\left\{N_{t} \log P\left(U_{t}-\theta\right)+\left(1-N_{t}\right) \log \left[1-P\left(U_{t}-\theta\right)\right]\right\} \\
& \quad-\gamma R\left(a_{0}, a_{1}, \ldots, a_{b}\right) .
\end{aligned}
$$

Expression (40) is now maximized for given $R$ and positive $\gamma$ where the role of $\gamma$ is to modify the relative importance of the penalty terms in the likelihood (40). The analyses presented

\footnotetext{
3 The package $R$ was used in the computational work of this section (CRAN 2008).
}

were based on the penalty function

$R=\sum_{u=0}^{b-1}\left(a_{u+1}-a_{u}\right)^{2}$.

The sandwich estimator was used to provide consistent estimates of the covariance matrix of the parameter estimates (Hilbe and Hardin 2001).

The following examples will illustrate the development of a sequence of models of increasing complexity progressing from single-input single-output models to multiple-input multiple-output models in which $a$ and $M$ in expression (37) will be taken to be vectors and $R$ will be the sum of the corresponding individual sum of the squared-differences of sequential terms.

\subsection{Single and multiple input with single-output at a fixed muscle length}

The first example illustrates, through the behaviour of the kernel functions, how the fusimotor inputs $\left(\gamma_{1}, \gamma_{2}\right)$ applied separately to the muscle spindle affect the responses of its secondary (II) and primary endings (Ia), and how these effects are altered when both inputs act simultaneously with the parent muscle held at a fixed length. The models used in the analysis of the single-input single-output experiment are

$$
\operatorname{Prob}\left\{\mathrm{II}_{t}=1 \mid \gamma_{1}\right\}=L\left(\sum_{u=0}^{b} a_{u} \gamma_{1, t-u}\right),
$$

$\operatorname{Prob}\left\{\mathrm{II}_{t}=1 \mid \gamma_{2}\right\}=L\left(\sum_{u=0}^{b} a_{u} \gamma_{2, t-u}\right)$,

$\operatorname{Prob}\left\{\operatorname{Ia}_{t}=1 \mid \gamma_{1}\right\}=L\left(\sum_{u=0}^{b} a_{u} \gamma_{1, t-u}\right)$,

$\operatorname{Prob}\left\{\mathrm{Ia}_{t}=1 \mid \gamma_{2}\right\}=L\left(\sum_{u=0}^{b} a_{u} \gamma_{2, t-u}\right)$,

where $L$ denotes the distribution function of the logistic distribution and the value of $b$ is taken to be 50. The models used in the analysis of the two-input single-output experiment are

$$
\begin{aligned}
& \operatorname{Prob}\left\{\mathrm{II}_{t}=1 \mid \gamma_{1}, \gamma_{2}\right\}=L\left(\sum_{u=0}^{b} a_{1, u} \gamma_{1, t-u}+\sum_{u=0}^{b} a_{2, u} \gamma_{2, t-u}\right), \\
& \operatorname{Prob}\left\{\mathrm{Ia}_{t}=1 \mid \gamma_{1}, \gamma_{2}\right\}=L\left(\sum_{u=0}^{b} a_{1, u} \gamma_{1, t-u}+\sum_{u=0}^{b} a_{2, u} \gamma_{2, t-u}\right) .
\end{aligned}
$$



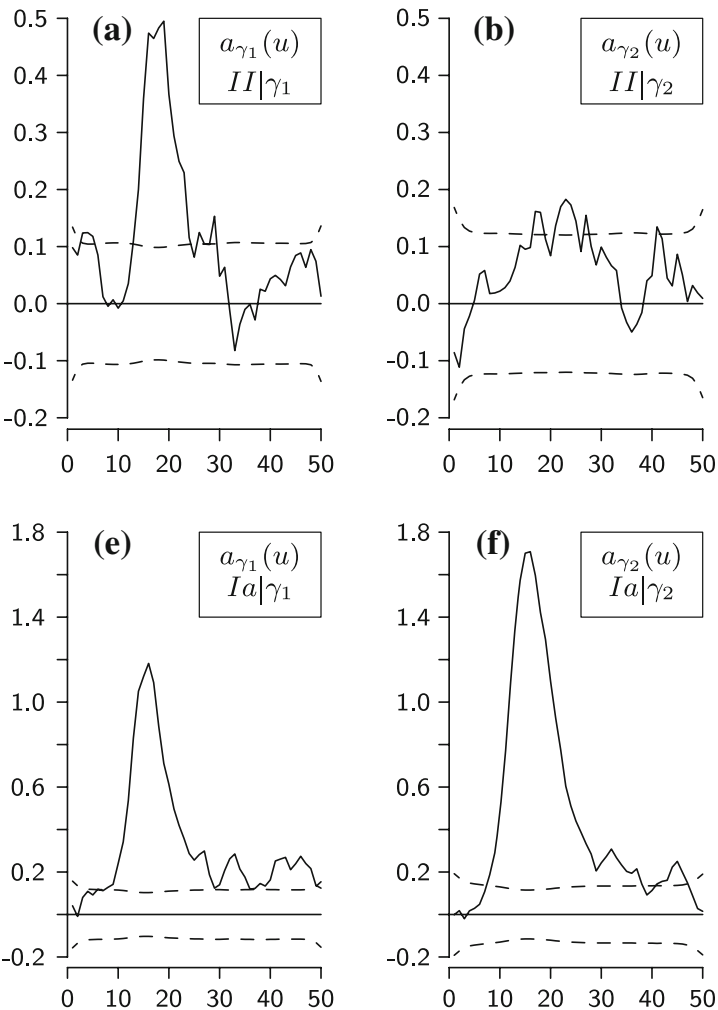

Fig. 5 Penalized maximum likelihood estimates of $a(u)$, the kernel functions (solid lines) for the models in Eqs. (41) and (42), together with twice their marginal standard error limits (dashed lines). $\mathbf{a}$ is the kernel function for the II response to $\gamma_{1}$, b is the kernel function for the II response to $\gamma_{2}, \mathbf{c}, \mathbf{d}$ are the kernel functions $a_{\gamma_{1}}$ and $a_{\gamma_{2}}$ for the

The kernel functions estimated from these models using a penalized log-likelihood procedure are illustrated in Fig. 5. It is clear from Fig. 5 (upper panels) that of the two fusimotor axons here, only $\gamma_{1}$ exerts an influence on the spindle secondary ending independently of whether or not both fusimotor axons act separately or together. On the other hand Fig. 5 (lower panels) illustrates that both fusimotor axons influence the primary ending. By contrast with the fusimotor effects on the secondary ending, a comparison of Fig. 5e with Fig. 5g and Fig. 5f with Fig. 5h indicates that when both inputs act simultaneously their individual influences are marginally reduced. It is also clear from Figure 5 that the $\gamma_{2}$ fusimotor axon affects only the primary ending whereas the $\gamma_{1}$ fusimotor axon influences both primary and secondary endings although its influence on the former is more pronounced. Furthermore, Fig. 2 (upper right panel) suggests that the effect of the linear model is improved when the action of both fusimotor axons are taken into account, and therefore it is anticipated that the kernel functions illustrated in Fig. 5c, $\mathrm{d}, \mathrm{g}$ and $\mathrm{h}$ provide more realistic representations of the effectiveness of fusimotor activity than those when each axon is stimulated independently since the former approaches the normal behaviour of the spindle.
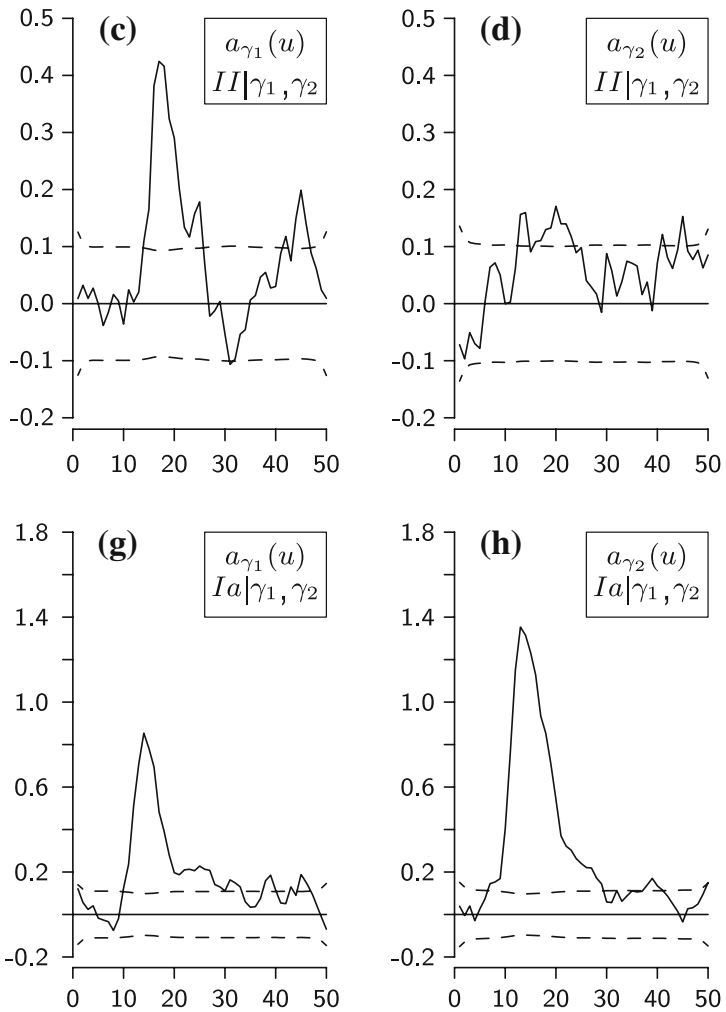

II response to $\gamma_{1}$ and $\gamma_{2}$. e is the kernel function for the Ia response to $\gamma_{1}, \mathbf{f}$ is the kernel function for the Ia response to $\gamma_{2}, \mathbf{g}, \mathbf{h}$ are the kernel functions $a_{\gamma_{1}}$ and $a_{\gamma_{2}}$ for the Ia response to $\gamma_{1}$ and $\gamma_{2}$. The horizontal scale gives lag $u$ in milliseconds

\subsection{Single and multiple input with single-output and random changes in muscle length}

The next example illustrates, through the behaviour of the kernel functions, how the fusimotor inputs $\left(\gamma_{1}, \gamma_{2}\right)$ applied simultaneously to the muscle spindle affect the responses of its secondary (II) and primary endings (Ia), and how these effects are altered in the presence of randomly varying length changes applied to the parent muscle. The object of this Section is to illustrate the sensitivity of the kernel functions with respect to random variations in the length of the parent muscle for the models defined by Eqs. (42).

Figure 6 illustrates clearly the sensitivity of kernel functions to imposed length changes. A comparison of Fig. $6 a, b$ with Fig. 6e, $\mathrm{f}$ indicates that the kernel functions $a_{\gamma_{1}}$ and $a_{\gamma_{2}}$, which characterise the response of the secondary ending to simultaneous fusimotor inputs, are largely unaltered by the presence of random variations in the length of the parent muscle. On the other hand the kernel function $a_{\gamma_{1}}$ which characterises the response of the primary ending (compare Fig. $6 \mathrm{c}$ and g) is no longer significant in the presence of random length changes, suggesting that the $\gamma_{1}$ fusimotor 

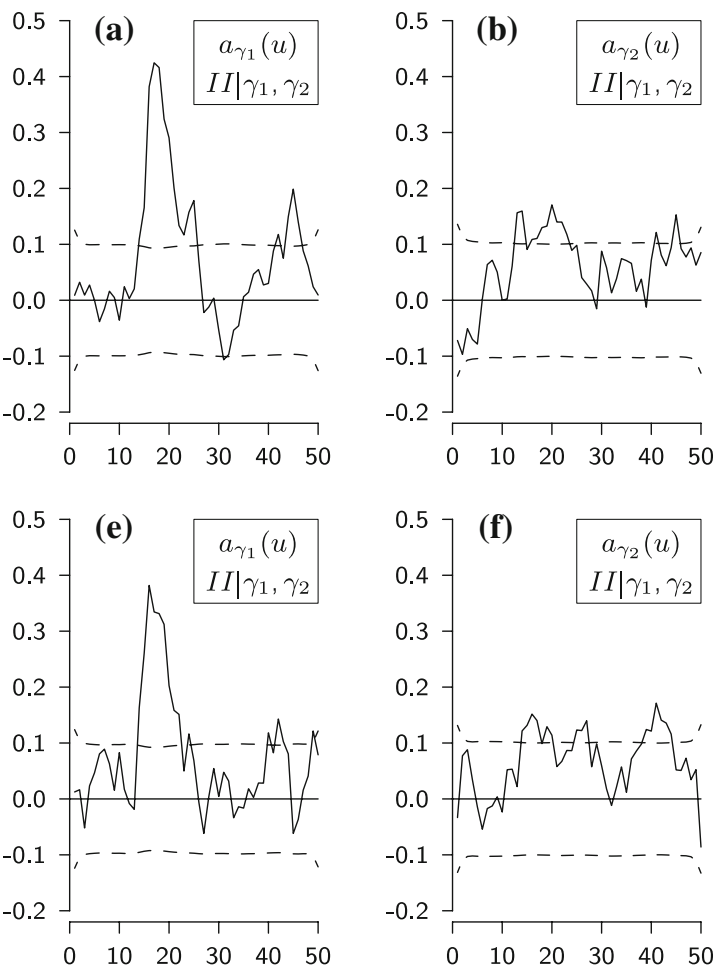

Fig. 6 Penalized maximum likelihood estimates of $a(u)$, the kernel functions (solid lines) for the models in Eqs. (41) and (42), together with twice their marginal standard error limits (dashed lines) at a fixed muscle length in $\mathbf{a}-\mathbf{d}$ and in the presence of random variations in the length of the parent muscle about this fixed length in $\mathbf{e}-\mathbf{h}$. Kernel functions for Ia and II responses are estimated when both fusimotor axons are stimulated. a and e show, respectively, the kernel function $a_{\gamma_{1}}$ for the II response at fixed length and for random length changes; $\mathbf{b}$ and $\mathbf{f}$ show,

axon influences only the secondary ending in the presence of random length changes. Furthermore, the kernel function $a_{\gamma_{2}}$ which characterises the response of the primary ending (compare Fig. 6d and h), although reduced considerably in magnitude in the presence of random length changes, nevertheless remains significant for a range of lags. In conclusion, randomly imposed length changes appear to uncouple the action of the $\gamma_{1}$ fusimotor axon on the response of the primary ending, whereas the same random length changes leave unchanged its effect on the response of the secondary ending. Therefore under dynamically imposed length changes, the response of the primary and secondary endings from the same muscle spindle at this level of analysis may provide two different kinds of information. The activity of the primary ending reflects the influence of fusimotor activity with the parent muscle held at a fixed length, but during dynamic length changes predominantly reflects the effect of these changes. On the other hand the response of the secondary ending reflects only the fusimotor activity and the fixed length about which these dynamic length changes have been imposed.
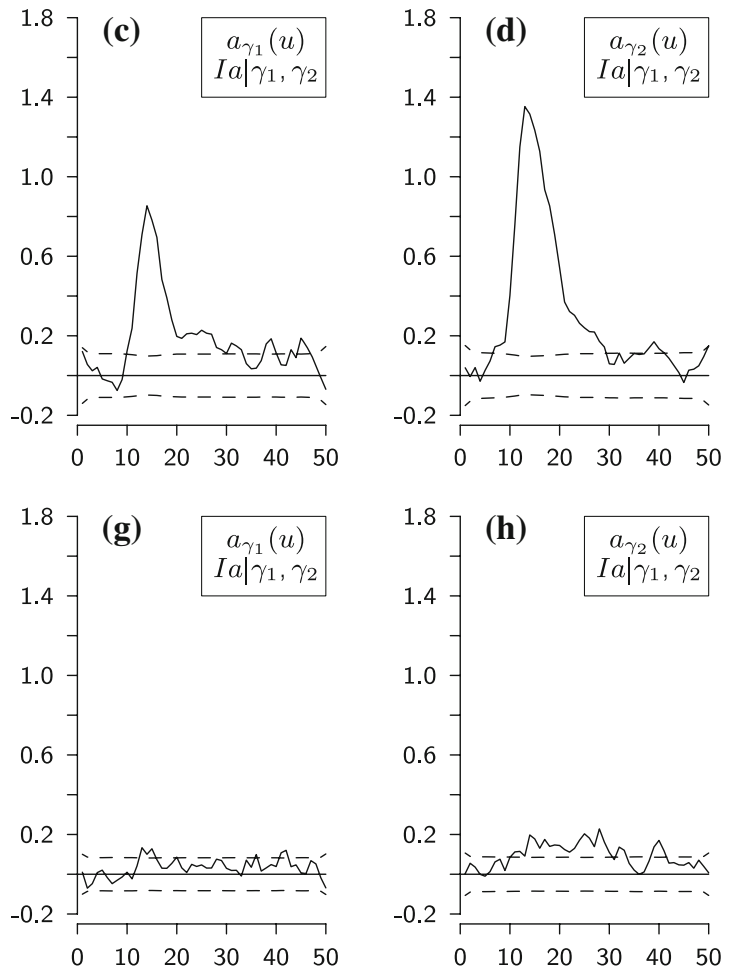

respectively, the kernel function $a_{\gamma_{2}}$ for the II response at fixed length and for random length changes; $\mathbf{c}$ and $\mathbf{g}$ show, respectively, the kernel function $a_{\gamma_{1}}$ for the Ia response at fixed length and for random length changes; $\mathbf{d}$ and $\mathbf{h}$ show, respectively, the kernel function $a_{\gamma_{2}}$ for the Ia response at fixed length and for random length changes. The horizontal scale gives lag $u$ in milliseconds. In this figure, $\mathbf{a}$ and $\mathbf{b}$ are taken from Fig. $5 \mathrm{c}$ and d, and $\mathbf{c}$ and $\mathbf{d}$ are taken from Fig. $5 \mathrm{~g}$ and $\mathrm{h}$

\subsection{Fusimotor and length-dependent interactions between responses of primary and secondary muscle spindle endings}

As in the previous Sections, changes in the behaviour of the kernel functions are used as an index of changes in the relationship between spike train discharges. In this Section, the changes in the kernel functions are used to examine how mutual interactions between the activity of Ia and II endings are influenced by fusimotor $\left(\gamma_{1}, \gamma_{2}\right)$ inputs when the parent muscle is held at fixed length and also when randomly varying length changes are applied to the parent muscle. The model used to investigate the mutual interactions between the activity of the primary and secondary muscle spindle endings in the absence of both length changes and fusimotor input is

$$
\begin{aligned}
& \operatorname{Prob}\left\{\mathrm{II}_{t}=1 \mid \mathrm{Ia}\right\}=L\left(\sum_{u=0}^{b} a_{u} \mathrm{Ia}_{t-u}\right), \\
& \operatorname{Prob}\left\{\mathrm{Ia}_{t}=1 \mid \mathrm{II}\right\}=L\left(\sum_{u=0}^{b} a_{u} \mathrm{II}_{t-u}\right) .
\end{aligned}
$$



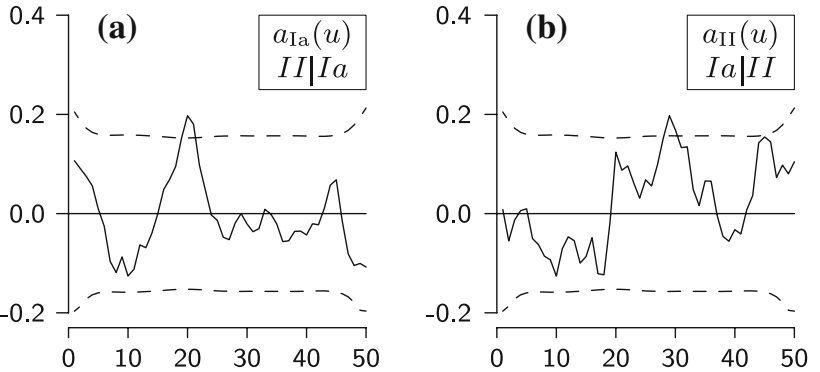

Fig. 7 Penalized maximum likelihood estimates of $a(u)$, the kernel functions (solid lines) for the models in Eqs. (43), together with twice their marginal standard error limits (dashed lines) at a fixed muscle length. The left panel shows the kernel functions for the II response when estimated from Ia activity, and the right panel shows the kernel functions for the Ia response when estimated from II activity. The horizontal scale gives lag $u$ in milliseconds

The kernel functions describing these interactions are illustrated in Fig. 7.

From Fig. 7 it is clear that there is negligible interaction between the activity of the primary and secondary endings in the absence of fusimotor activity and changes in muscle length. In a previous study Rosenberg et al. (1989, see Fig. 6) have demonstrated that the activity of the primary and secondary endings become correlated in the presence of either fusimotor activity or random changes in muscle length. In the former the correlation occurs at frequencies below $20 \mathrm{~Hz}$, whereas in the latter the correlation occurs at frequencies between 20 and $60 \mathrm{~Hz}$.

Fig. 8 Penalized maximum likelihood estimates of $a(u)$, the kernel functions (solid lines) for the model in Eq. (44), together with twice their marginal standard error limits (dashed lines) at a fixed muscle length in $\mathbf{a}-\mathbf{c}$ and in the presence of random variations in the length of the parent muscle about this fixed length in $\mathbf{d}-\mathbf{f}$. Kernel functions for the II response are estimated when both fusimotor axons are stimulated and the Ia activity is taken into account. a and $\mathbf{d}$ show, respectively, the kernel function $a_{\gamma_{1}}$ for the II response at fixed length and for random length changes; $\mathbf{b}$ and $\mathbf{e}$ show, respectively, the kernel function $a_{\gamma_{2}}$ for the II response at fixed length and for random length changes; $\mathbf{c}$ and $\mathbf{f}$ show, respectively, the kernel function $a_{\text {Ia }}$ for the II response at fixed length and for random length changes. The horizontal scale gives lag $u$ in milliseconds
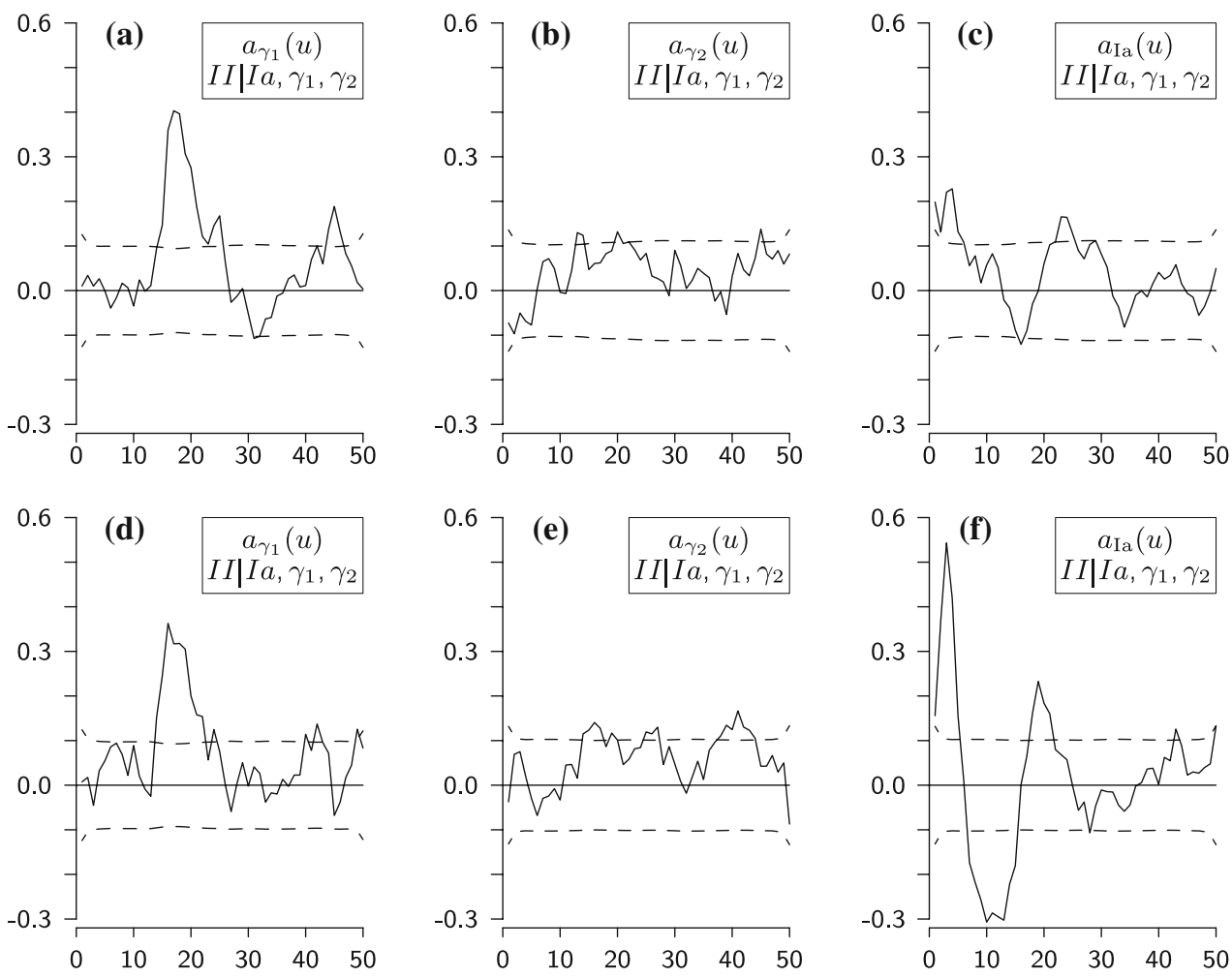

Equations (43) are now adapted to take account of the influence of fusimotor input and imposed random length changes to obtain the extended model

$$
\begin{aligned}
& \operatorname{Prob}\left\{\mathrm{II}_{t}=1 \mid \gamma_{1}, \gamma_{2}, \mathrm{Ia}\right\} \\
& \quad=L\left(\sum_{u=0}^{b} a_{1, u} \gamma_{1, t-u}+\sum_{u=0}^{b} a_{2, u} \gamma_{2, t-u}+\sum_{u=0}^{b} a_{\mathrm{Ia}, u} \mathrm{Ia}_{t-u}\right),
\end{aligned}
$$

$\operatorname{Prob}\left\{\mathrm{Ia}_{t}=1 \mid \gamma_{1}, \gamma_{2}, \mathrm{II}\right\}$

$$
=L\left(\sum_{u=0}^{b} a_{1, u} \gamma_{1, t-u}+\sum_{u=0}^{b} a_{2, u} \gamma_{2, t-u}+\sum_{u=0}^{b} a_{\mathrm{II}, u} \mathrm{II}_{t-u}\right) \text {. }
$$

The interactions characterised by each model equation are explored separately to assess whether or not there is a directional component to the interaction between the discharges of primary and secondary endings.

Figure 8 illustrates the influence of Ia, $\gamma_{1}, \gamma_{2}$ activity on the response of the secondary ending. The upper row of this Figure demonstrates that the dominant factor correlating II with Ia is the $\gamma_{1}$ activity (Fig. 8a), although there may be a small contribution from Ia activity (Fig. 8c). In the presence of a randomly varying length change the influence of $\gamma_{1}$ activity on II remains largely unchanged (Fig. 8d), whereas that of Ia activity on II is enhanced (Fig. 8f). The analysis continues by considering how the response of the primary ending 

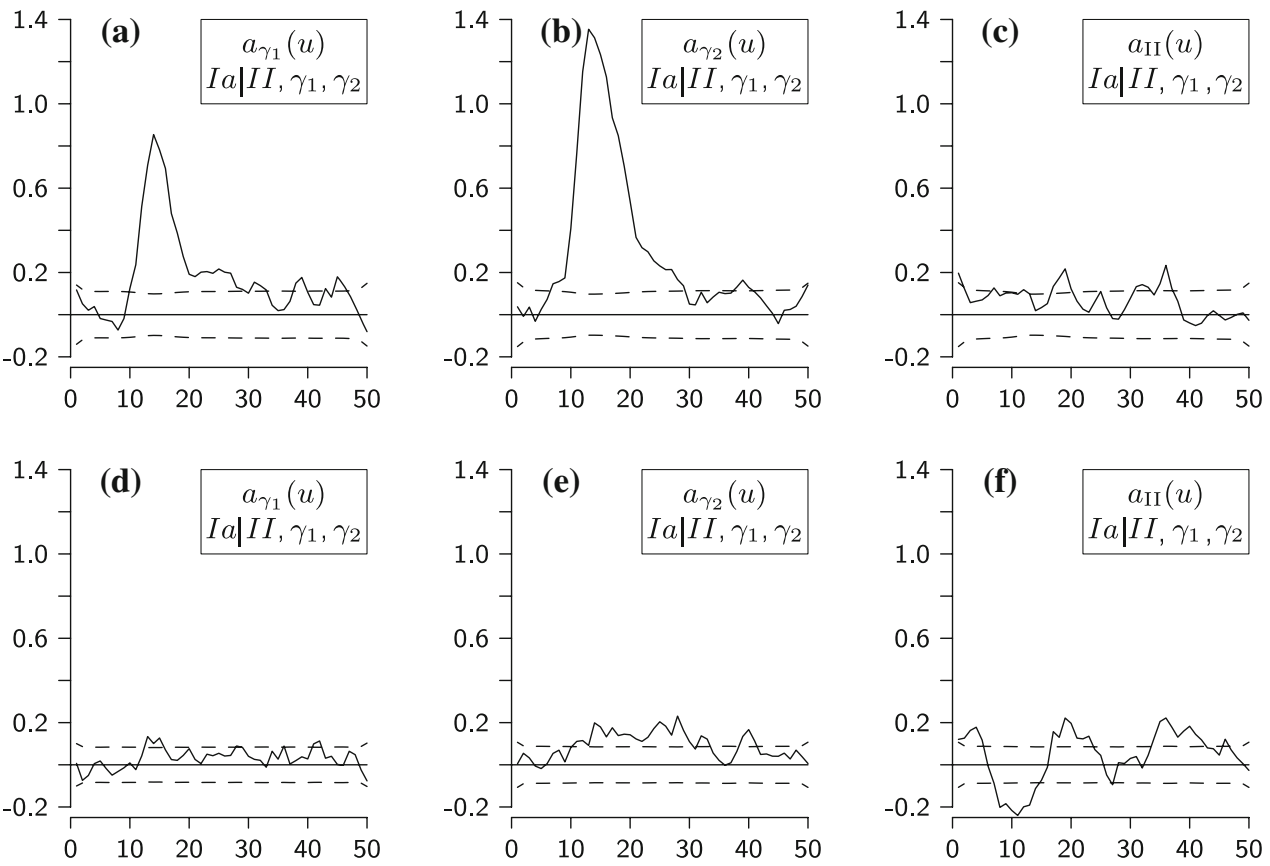

Fig. 9 Penalized maximum likelihood estimates of $a(u)$, the kernel functions (solid lines) for the model in Eq. (45), together with twice their marginal standard error limits (dashed lines) at a fixed muscle length in $\mathbf{a}-\mathbf{c}$ and in the presence of random variations in the length of the parent muscle about this fixed length in $\mathbf{d}-\mathbf{f}$. Kernel functions for the Ia response are estimated when both fusimotor axons are stimulated and the II activity is taken into account. $\mathbf{a}$ and $\mathbf{d}$ show, respectively, the kernel function $a_{\gamma_{1}}$ for the Ia response at fixed length and for random length changes; $\mathbf{b}$ and $\mathbf{e}$ show, respectively, the kernel function $a_{\gamma_{2}}$ for the Ia response at fixed length and for random length changes; $\mathbf{c}$ and $\mathbf{f}$ show, respectively, the kernel function $a_{\mathrm{II}}$ for the Ia response at fixed length and for random length changes. The horizontal scale gives lag $u$ in milliseconds depends of the properties of the activity of the processes II, $\gamma_{1}$ and $\gamma_{2}$. These results are illustrated in Fig. 9 .

By contrast with the interaction from Ia onto II, where the predominant influence is due to the activity of the $\gamma_{1}$ fusimotor axon with a small influence from Ia activity, for the interaction in the direction from II onto Ia in the absence of random length changes, the activity of both $\gamma_{1}$ and $\gamma_{2}$ fusimotor axons contribute to the correlation between the responses of the primary and secondary endings (Fig. 9a, b), with no contribution arising from the activity of the secondary ending (Fig. 9c). On the other hand, in the presence of a random length change, the contribution from $\gamma_{1}$ to Ia is suppressed, the contribution from $\gamma_{2}$ to Ia is greatly reduced and the contribution for II to Ia appears to be unchanged.

The interaction between the Ia and II processes will be interpreted using the model equations (44) and (45). In each of these models the argument of the link function $L$ contains three components. Each argument includes contributions from the activities of $\gamma_{1}$ and $\gamma_{2}$ with the argument in model (44) including a contribution from Ia activity and the argument of model (45) including a contribution from II activity. Consider the Ia spike train with an identical line of reasoning for the II spike train. The Ia spike train may be interpreted as resulting from the combined effect of the $\gamma_{1}$ activity, the $\gamma_{2}$ activity and the activity of unknown processes resulting from the behaviour of the length of the parent muscle. Since the activity of the $\gamma_{1}$ and $\gamma_{2}$ processes are included in the argument of the link function as separate components, the role of the II spike train in this argument can be thought of as a proxy for the contribution of these unknown processes.

With the Ia and II processes interpreted as proxies for unknown processes, the question now becomes one of whether or not there is a preferred direction of interaction between these processes contingent on the linear models chosen. Consider first the case of the parent muscle held at a fixed length. In models (44) and (45), the unknown processes represented by their respective kernel functions (Figs. 8c and 9c) make negligible contributions to the argument of their respective link functions and so to the probability of the occurrence of a Ia or II spike. On the other hand, the $\gamma_{1}$ activity makes a significant contribution to both link functions and therefore to the probability of the occurrence of a Ia or II spike (Figs. 8a and 9a). Finally, $\gamma_{2}$ activity contributes only to the link function of model (45) and thus to the probability of the occurrence of a Ia spike (Figs. 8b and 9b).

In the presence of dynamically imposed length changes, the relative contributions of each component of the argument of the link function are altered. First, the activity of $\gamma_{1}$ contributes only to the argument of the link function in model 
(44) (Figs. 8d and 9d), whereas previously in the case of a fixed length the activity of $\gamma_{1}$ contributed to the argument of both link functions (Figs. 8a and 9a). Second, the contribution of the activity of $\gamma_{2}$ to the argument of the link function in model (44) remains unchanged in the presence of dynamically imposed length changes (Fig. 8b, e), whereas its contribution to the argument of the link function in model (45) is greatly reduced (Fig. 9b, e). Finally, the most dramatic change in the argument of the link functions occurs in the contributions made by the unknown processes represented by Ia activity in the link function of model (44). In particular note the striking difference that the unknown processes associated with the Ia activity have on the II response in the absence and in the presence of a dynamic length changed imposed on the parent muscle (compare Fig. 8c with Fig. 8f). Therefore in the presence of dynamically imposed length changes, the lower panels of Figs. 8 and 9 demonstrate that the unknown processes associated with Ia activity exert a stronger influence on the probability of the occurrence of a II spike than the unknown processes associated with II activity have on the probability of occurrence of a Ia spike. These results seem to suggest that under dynamic length changes the activity of the Ia and II processes carry different information about the effects of the inputs imposed on the muscle spindle.

\section{Concluding remarks}

A frequency domain approach and a time domain approach have been combined in an investigation of the behaviour of the primary and secondary endings of an isolated muscle spindle in response to the activity of two static fusimotor axons when the parent muscle is held at a fixed length and when it is subjected to random length changes.

The frequency domain analysis is based on a stationary input with the assumption referring only to the first and second order moments of the processes, and has been used to examine the properties of the muscle spindle by providing a measure of the error process associated with the linear model, and to specify how the interactions between the recorded processes contribute to this process. On the other hand the time domain approach involves a full probability model in which the inputs can be general processes which need not be stationary processes - a very important feature of this approach since in many experimental situations the input processes are not stationary. The construction of the time domain model allows new input processes to be included directly to the model in arbitrary combinations with existing input processes. A number of mathematical models of the muscle spindle have been developed (Mileusnic et al. 2006). One application of the procedures developed in this article would be to apply them to assess the linear behaviour of these models using the frequency domain approach outlined in Sects. 3 and 3.2, and to examine the behaviour of the kernels derived from the time domain methods presented in Sect. 4.

The main feature of the time domain results is that they illustrate how the addition of further processes to the input interact with the effects of existing inputs. This property of the time domain approach was used to identify a preferred direction of interaction between the processes underlying the generation of the activity of the primary and secondary endings. By contrast, the frequency domain approach showed that the linear range of the model is extended when both input processes are present. By associating an error process with the linear model, it was shown that this model is reasonable at frequencies below $25 \mathrm{~Hz}$ in the absence of an imposed length change. The range of frequencies for which the model is adequate lie within the physiological range of naturally occurring frequencies at fixed lengths. On the other hand, in the presence of dynamic variations in the length of the parent muscle, the linear model did not give a satisfactory description of the behaviour of the input and output processes. However, this description may be improved by taking account of nonlinear behaviour in the manner proposed by Moore and Auriemma (1985). The results presented in this work emphasise that the analysis of the behaviour of complex systems benefits from a combination of frequency and time domain methods.

Acknowledgments D. R. Brillinger acknowledges support from National Science Foundation grant DMS-0707157. D.R. Brillinger's work in this paper is dedicated to Raquel and Charles and Jef who brought Pepe and him so close together.

Open Access This article is distributed under the terms of the Creative Commons Attribution Noncommercial License which permits any noncommercial use, distribution, and reproduction in any medium, provided the original author(s) and source are credited.

\section{References}

Banks RW, Barker D, Stacy MJ (1981) Structural aspects of fusimotor effects on spindle sensitivity. In: Taylor A, Procazka A (eds) Muscle receptors in movement. Macmillan Publishers Ltd., London, pp 5-16

Boyd IA (1981) The action of the three types of intrafusal fibre in isolated cat muscle spindles of the dynamic and length sensitivities of primary and secondary endings. In: Taylor A, Procazka A (eds) Muscle receptors in movement. Macmillan Publishers Ltd., London, pp 17-32

Brillinger DR (1975a) The identification of point process systems. Ann Probab 3:909-924

Brillinger DR (1975b) Time series: data analysis and theory. Holt Rinehart Winston, New York

Brillinger DR (1983) The finite Fourier transform of a stationary process. In: Brillinger DR, Krishnaiah PR (eds) Handbook of Statistics, vol 3. Academic Press, New York, pp 21-37

Brillinger DR (1992) Nerve cell spike train data analysis: a progression of techniques. J Am Stat Assoc 87:260-271

Brillinger DR, Bryant HL Jr, Segundo JP (1976) Identification of synaptic interactions. Biol Cybern 22:213-228 
Brillinger DR, Segundo JP (1979) Empirical examination of the threshold model of neuron firing. Biol Cybern 35:213-220

Cox DR, Marmarelis PZ, Segundo JP, Brillinger DR (1975) The identification of point process systems: discussion of Professor Brillinger's paper. Ann Probab 3:924-929

CRAN: Comprehensive R Archive Network (2008). http://www. r-project.org

Edgley SA, Jankowska E (1987) An interneuronal relay for group I and II muscle afferents in the midlumbar segments of the cat spinal cord. J Physiol 389:647-674

Gladden MH, Matsuzaki H (2002) Static $\gamma$-motoneurons couple group Ia and II afferents of single muscle spindles in anaesthetised and decerebrate cats. J Physiol London 54:273-288

Halliday DM, Gladden MH, Rosenberg JR (1987) Fusimotor induced phase differences between primary and secondary endings from the same muscle spindle. In: Hník P, Soukup T, Vejsada R, Zelená (eds) Mechanoreceptors-development, structure and function. Springer, Berlin, pp 225-230

Hilbe M, Hardin JN (2001) Generalized linear models and extensions. Stata Press, College Station

Jankowska E (1992) Interneuronal relay in spinal pathways from proprioceptors. Prog Neurobiol 38:335-378
Jankowska E, Hammar I (2002) Spinal interneurones: how can studies in animals contribute to the understanding of spinal interneuronal systems in man? Brain Res Rev 40:19-28

Lindsay KA, Ogden JM, Rosenberg JR (2001) Advanced numerical methods for modelling dendrites. In: Roman R. Poznanski (ed) Biophysical neural networks. Mary Ann Liebert Inc., New York, pp 411-493

McCullagh P, Nelder JA (1989) Generalized linear models, 2nd edn. Chapman and Hall, Glasgow

Mileusnic MP, Brown IE, Lan N, Loeb GE (2006) Mathematical models of proprioceptors. I. Control and transduction in the muscle spindle. J Neuro Physiol 96:1772-1788

Moore GP, Auriemma RA (1985) Prediction of muscle stretch receptor behaviour using Wiener kernels. Brain Res 331:185-189

Rosenberg JR, Amjad AM, Breeze P, Brillinger DR, Halliday DM (1989) The Fourier approach to the identification of functional coupling between neuronal spike trains. Prog Biophys Mol Biol 53:1-31

Vapnik V (1982) Estimation of dependencies based on empirical dataempirical inference science. Springer, New York 